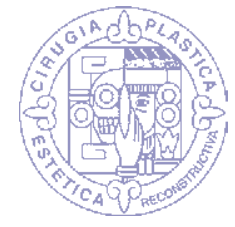

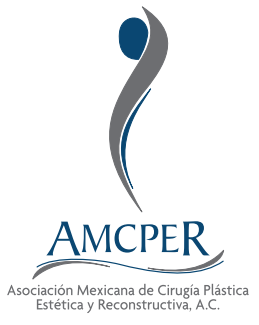

\title{
Utilidad clínica de la zetaplastia de cuatro colgajos en secuelas de quemadura
}

\author{
Clinical utility of four flaps Z-plasty in burns scar contracture
}

\author{
Dr. José Luis Pérez-Ávalos,* Dra. Virginia Núñez-Luna ${ }^{\ddagger}$
}

Palabras clave: Bridas postquemadura, zetaplastia de cuatro colgajos, secuelas de quemaduras.

Keywords: Post-burn contractures, four flap zetaplasty, post-burn sequels.

* Médico adscrito a la Unidad de Quemados del Hospital Materno Pediátrico Xochimilco. Profesor en la Universidad Autónoma Metropolitana Xochimilco, México.

¥ Jefa del Servicio de la Unidad de Quemados, Hospital Materno Pediátrico Xochimilco. Profesora en la Universidad Autónoma Metropolitana Xochimilco, México.

Los autores de este artículo no tienen conflicto de intereses qué declarar.

Recibido:

02 julio 2018 Aceptado para publicar: 01 junio 2019

\section{RESUMEN}

Las lesiones por quemadura son frecuentes. Sus secuelas constituyen retos constantes para el equipo de tratamiento, pues nos enfrentamos a retos de toda índole en cualquier parte del cuerpo en la que exista alguna alteración en su estructura, función o estética. Presentamos nuestra experiencia en la liberación de bridas como secuelas de quemaduras en niños en un hospital pediátrico de tipo asistencial de la Ciudad de México. Para ello, utilizamos la zetaplastia de cuatro colgajos; analizamos la indicación, planeación, ejecución y resultados de casos clínicos, así como las ventajas y desventajas de dicha técnica.

\section{ABSTRACT}

Burn injuries are frequent and their consequences are a constant challenge for the treatment team. We face all kinds of challenges in any part of the body where there is some alteration in its structure, function or aesthetics. We present our experience in the release of scar contractures as sequelae of burns in the pediatric population in a Public Pediatric Assistance Hospital in Mexico City, using the four flap zetaplasty, analyzing the indication, planning, execution and results of clinical cases, as well as the advantages and disadvantages of the given technique.

\section{INTRODUCCIÓN}

$\mathrm{S}_{\mathrm{s}}^{\mathrm{e}}$ esabe desde hace tiempo que la frecuencia de las lesiones por quemadura en nuestro país es cada vez más importante. Por ejemplo, en el año 2008, llegó a ocupar el vigésimo lugar entre las causas de enfermedad, ${ }^{1}$ con una tasa de incidencia de 110 casos por 100 mil habitantes. Por consiguiente, las secuelas que se presentan en esta patología son necesariamente numerosas y constituyen un reto constante, tanto para el equipo de terapia y rehabilitación como para los cirujanos plásticos que atendemos esta patología. ${ }^{2}$ En efecto, nuestra especialidad se enfrenta a retos o secuelas de todo tipo y en cualquier parte del cuerpo donde haya ocurrido alguna alteración en la estructura, en la función y en el aspecto estético.

El manejo de las secuelas en un paciente quemado no se limita a un solo tipo de procedimiento quirúrgico; es decir, no termina con los llamados aseos quirúrgicos o con la toma de injertos en la fase aguda o subaguda, los cuales están destinados a salvar la vida a los enfermos. Las modalidades de técnicas de reparación van desde la colocación de un injerto de espesor parcial o total para un cambio de cubierta cutánea, aplicado como una unidad o subunidad estética, ${ }^{3}$ la rotación de colgajos para dar cubierta a alguna estructura vital expuesta como hueso, tendones, vasos, cartílago, etc., el empleo de la expansión tisular para cambiar la cubierta cutánea que ofrezca una mejor cobertura y aspecto estético, hasta la cubierta con colgajos a distancia con técnicas microquirúrgicas; todas ellas parte del armamentario que utilizamos para corregir las secuelas de lesiones tan severas que pueden ocurrir por este tipo de patología, lo que conocemos como escala reconstructiva. ${ }^{4}$

Por ello, en muchos casos tenemos a pacientes con bridas o cicatrices retráctiles que condicionan importantes limitaciones desde el punto de vista funcional, que repercuten también en el aspecto estético y en la psique 
Tabla 1: Tipos de zetaplastia de acuerdo con el ángulo y su ganancia tisular.
Tipo de zetaplastia (ángulo)
30 grados
45 grados
50
60 grados
75
75 grados
100
90 grados
25
en porcentaje

de los pacientes. ${ }^{5}$ En la mayor parte de los casos, esas alteraciones cicatriciales cruzan, por así decir, superficies articulares, lo que limita la extensión de algún segmento corporal, por lo que es necesario ofrecer soluciones desde el punto de vista quirúrgico para liberar las bridas.

Como parte de las denominadas plastias locales tenemos, por ejemplo, colgajos de rotación, de avance, colgajos tipo al azar (random), colgajos fasciocutáneos y también, descritas desde hace tiempo, una serie de técnicas en las que se intercambian colgajos, como las zetaplastias. Una de las primeras descripciones de esta técnica data de 1854, con Denonvilliers; ${ }^{6}$ desde entonces se han descrito claramente y utilizado por diversos e importantes autores de nuestra especialidad, ${ }^{7}$ como Mustardé, Fuente del Campo y Trigos, para la corrección quirúrgica del epicanto con zetaplastias de cuatro colgajos, ${ }^{8}$ pasando por las denominadas W-plastias.

El objetivo de este trabajo es revisar y proponer lo que en nuestra experiencia constituye una técnica fundamental en el quehacer quirúrgico cotidiano que se conoce como zetaplastia de cuatro colgajos, basada en los principios de Mir y Mir, ${ }^{9}$ mencionando las principales ventajas en su realización.
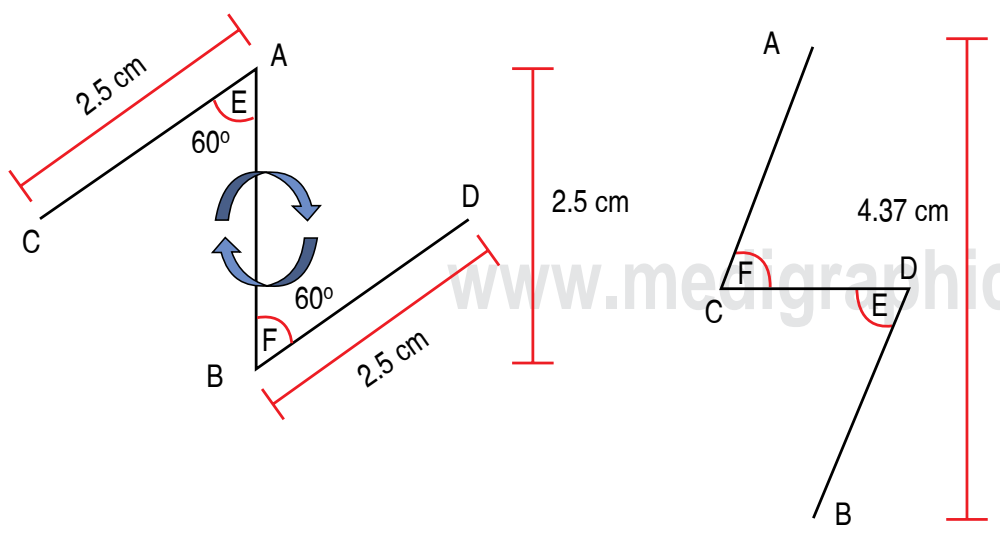

Figura 1: Zetaplastia de dos colgajos.

\section{Diseño y ganancia de tejido de las zetaplastias}

En la Tabla 1 reproducimos en porcentaje la ganancia tisular de los diferentes tipos de zetaplastia, de acuerdo con sus ángulos internos. Se sabe que, entre mayor sea el ángulo, mayor será la ganancia en longitud del tejido escindido, sobre todo en la piel, el cual dependerá también de las llamadas propiedades viscoelásticas de ésta. También es cierto que, a mayor amplitud en el ángulo, deberá ser mayor el despegamiento (socavamiento) y se incrementa el riesgo de lesionar tejidos circunvecinos, detalle que se debe tener muy en cuenta.

En términos generales, se aconseja que todas las ramas de la zetaplastia deben tener la misma longitud y el mismo ángulo, para que al momento de intercambiar los colgajos, éstos coincidan perfectamente. Esta regla no se aplica a las Ilamadas zetaplastias de ramas asimétricas, cuyo ajuste dependerá, por un lado, del despegamiento cutáneo y, por el otro, de las propiedades físicas cutáneas.

Se sugiere también que en su diseño se trace primero la brida a liberar; es decir, la línea o rama central motivo de la cirugía. Esto es importante, sobre todo en las bridas postquemadura, ya que determinará el máximo beneficio quirúrgico (Figuras 1 y 2).

\section{CASOS CLÍNICOS}

\section{Caso 1}

Se trata de una niña de cuatro años, con antecedente de quemadura a los 2 años, quien ameritó internamiento y manejo quirúrgico de las zonas de quemadura en el brazo derecho con toma y aplicación de injertos de piel, desarrollando como secuelas brida en pliegue del codo y limitación para la extensión. Se le 
1.

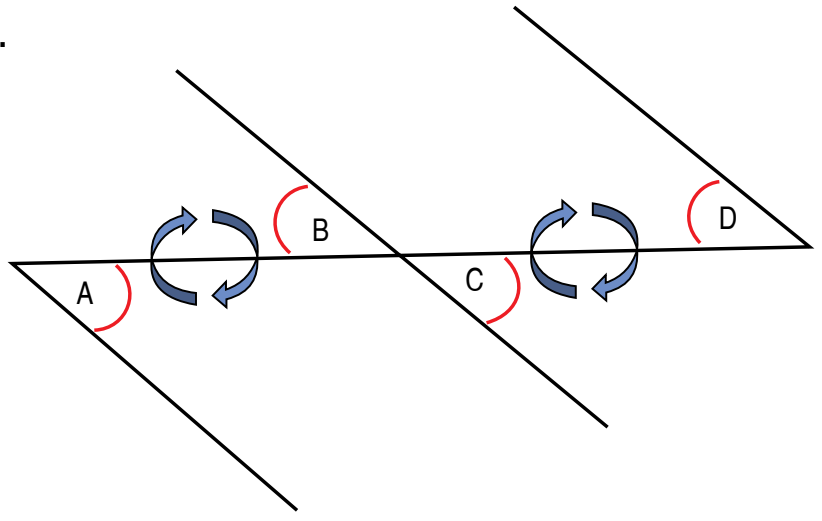

2.

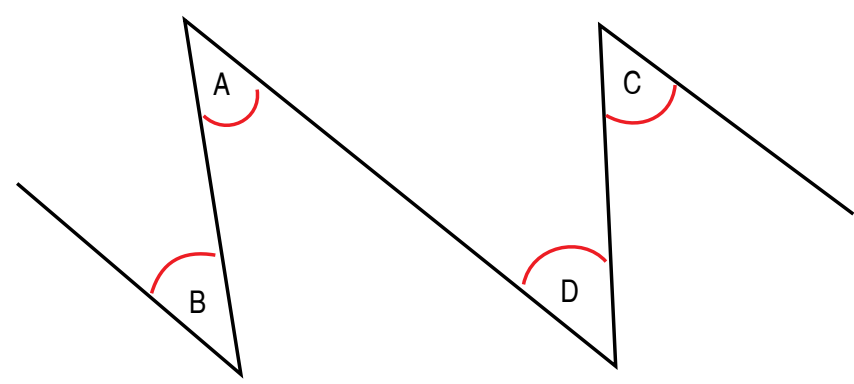

Figura 2: Zetaplastia de cuatro colgajos.

realizó una zetaplastia de cuatro colgajos a 45 grados (Figura 3), consiguiendo la liberación total de la brida sin complicaciones y, con ello, la extensión completa de dicha articulación (Figura 4).

\section{Caso 2}

Se presentó en nuestro hospital una niña de tres años, con antecedente de quemadura de segundo grado profundo al año de edad, con afectación del dorso del brazo y mano derechos, con manejo conservador y sin empleo de férula funcional, con el objetivo de evitar secuelas; esto ocasionó cicatriz retráctil en el dorso de la mano, afectando el primero, tercero y cuarto dedo. Se programó para liberación de la brida de tercero y cuarto dedo, en conjunto con una zetaplastia de cuatro colgajos a 75 grados (Figura 5), logrando una corrección parcial de la deformidad, con liberación incompleta de la brida a nivel de la articulación metacarpofalángica (Figura 6).

\section{Caso 3}

Presentamos el caso de un niño de siete años, con antecedente de quemadura severa en el $70 \%$ de la superficie corporal, quien fue trasladado a Galveston, en donde se le aplicaron injertos en mallas para cubrir la mayor parte de las quemaduras en la etapa aguda. A pesar del empleo de férulas de soporte para prevenir contracturas, desarrolló bridas en las regiones axilares, lo que condicionó la limitación a la extensión-abducción de la articulación del

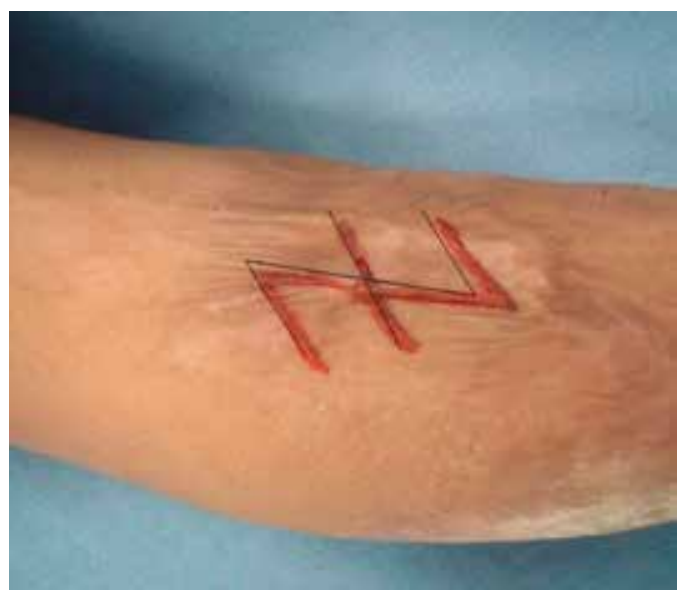

Figura 3: Diseño de zetaplastia de cuatro colgajos a 45 grados en un paciente pediátrico con secuelas de quemadura.

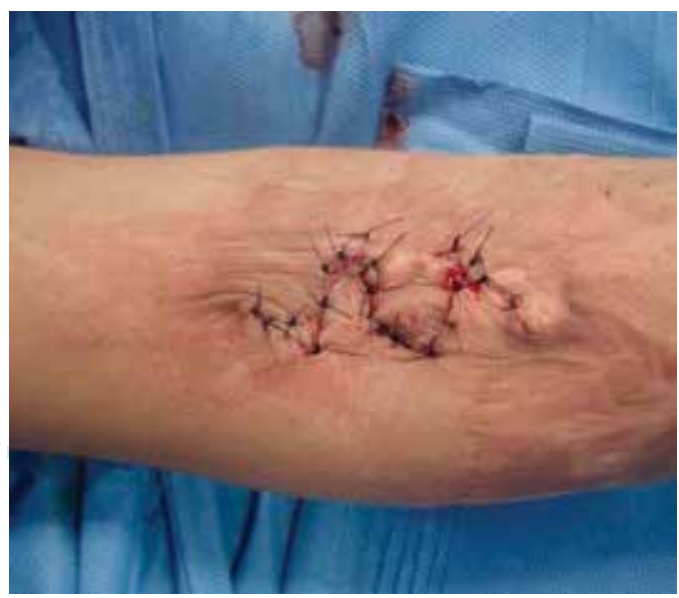

Figura 4: Resultante de zetaplastia de cuatro colgajos en brida de región anterior del codo en un paciente pediátrico con secuelas de quemadura. 


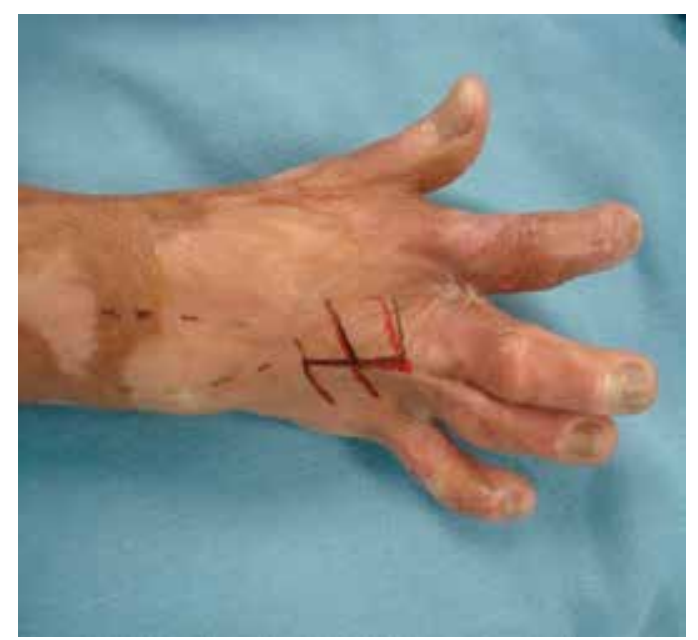

Figura 5: Diseño de zetaplastia de cuatro colgajos con ángulos a 75 grados en brida de dorso de la mano de un paciente pediátrico con secuelas de quemadura.

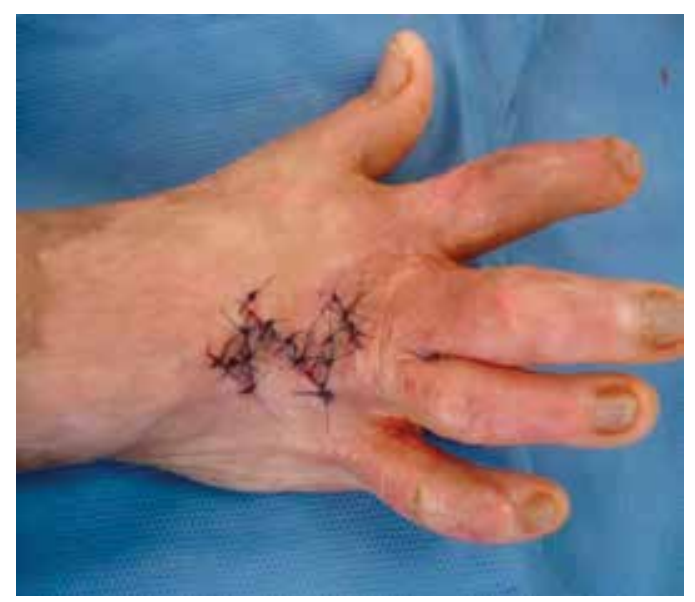

Figura 6: Resultante de zetaplastia en un paciente con secuelas de quemadura.

hombro. Se programó y realizó una zetaplastia de cuatro colgajos con ángulo a 45 grados (Figura 7), logrando la liberación total de la brida, teniendo como complicación necrosis parcial del vértice del colgajo distal, de la cual se recuperó con cierre por segunda intención (Figura 8).

\section{RESULTADOS}

En los casos 1 y 3 se logró una corrección total de la limitación de movimiento de las articula- ciones afectadas por la contractura. En ambos casos, se requirió del apoyo de la unidad de rehabilitación para complementar los resultados logrados con la cirugía. En el caso 3, hubo una zona de necrosis parcial en el colgajo distal debido probablemente al adelgazamiento del colgajo en el momento de su levantamiento. El paciente ameritó sólo vigilancia y curaciones, y no hubo necesidad de reintervenirlo por haber cerrado por segunda intención, sin que ello alterara el buen resultado.

En el caso 2 sólo se logró una corrección parcial de la deformidad debido a los severos cambios articulares, secuelas de un inadecuado

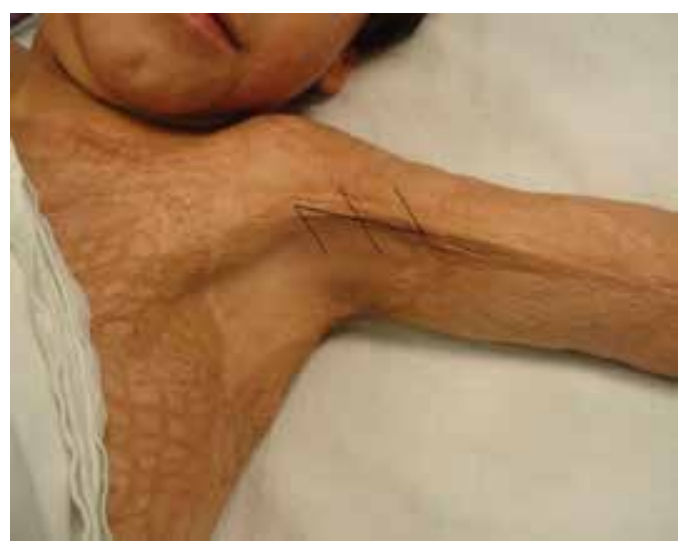

Figura 7: Diseño de zetaplastia de cuatro colgajos a 45 grados en brida de región axilar en un paciente pediátrico con secuelas de quemadura.

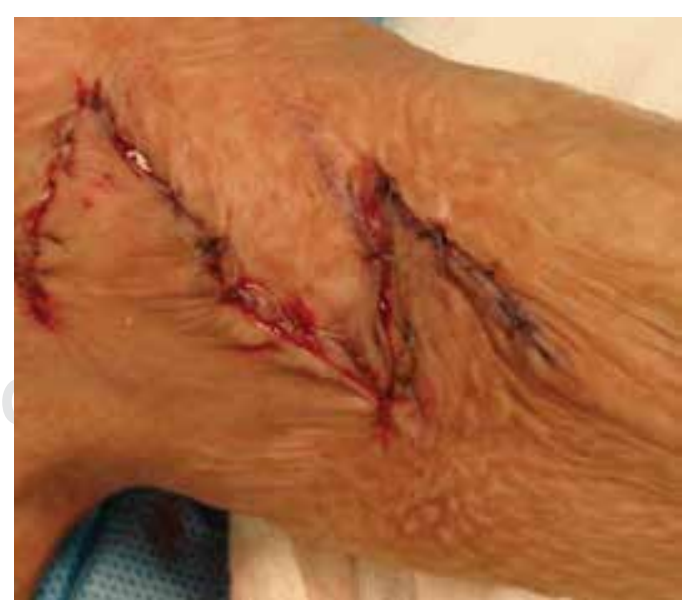

Figura 8: Resultado postoperatorio de zetaplastia para liberación de brida en región axilar. 
programa de ferulización y terapia física. La liberación cutánea no fue suficiente y creemos que también estuvo limitada por el fino grosor de la piel del dorso de la mano y por la proximidad y fijación a los elementos tendinosos (vaina y epitenón) de los tendones extensores y, obviamente, a los cambios anatómicos ocasionados por la quemadura. La paciente desarrolla en la actualidad un intenso programa de rehabilitación y ferulización dinámica para corregir las alteraciones articulares.

\section{DISCUSIÓN}

Las secuelas en los casos de pacientes con quemaduras son muy frecuentes, aun cuando se cuente en la actualidad con medidas preventivas para minimizarlas. En nuestro país, no son suficientes los profesionales o personal entrenado para tal fin, por lo que cabe esperar que nos sigamos enfrentando a esta patología. En cuanto a su localización anatómica, se ha reportado que las deformidades por contractura son más frecuentes en las extremidades $(43 \%)$, cuello $(30 \%)$, cara $(20 \%)$, tronco y otras zonas $(7 \%) .{ }^{10}$

Dentro de las medidas preventivas que han tenido buenos resultados podemos mencionar el uso de férulas anatómicas, ${ }^{2}$ que evitan la llamada "posición de confort", que sabemos dará paso a una deformidad. La terapia física desde la fase aguda de la quemadura ayudará también a evitar las rigideces articulares propias del paciente quemado. ${ }^{11}$ En las siguientes fases, la epitelización o postinjerto, la utilización de mallas para presoterapia, el empleo tópico de agentes para control de la cicatriz y el uso de láminas de silicón ayudarán a prevenir o corregir las cicatrices hipertróficas o queloides, para así lograr una mejoría funcional y estética.

En cuanto a los procedimientos quirúrgicos, ya mencionamos los que están indicados de acuerdo con la complejidad de cada caso y con la escala reconstructiva. ${ }^{4}$ Diversos autores refieren que la mayoría de los casos se resuelven con las llamadas plastias locales, por lo que no debemos minimizar su importancia terapéutica.

Dentro de este grupo de plastias locales, las zetaplastias están consideradas como la base de la terapéutica quirúrgica hasta nuestros días, 5,12 con excelentes resultados.
Hemos reunido alguna experiencia clínica con la utilización de la zetaplastia de cuatro colgajos; el empleo de esta técnica nos ha permitido resolver una gran cantidad de casos de secuelas de quemadura con las siguientes ventajas.

El diseño de la zetaplastia de cuatro colgajos es fácil y nos ahorra tiempo quirúrgico; en el caso de los pacientes pediátricos, nos exime de realizar grandes despegamientos al levantar los colgajos, evitando con esto, por un lado, el sangrado importante $y$, por el otro, minimiza el riesgo de dañar estructuras anatómicas adyacentes; esto es muy importante en segmentos anatómicos por donde discurren estructuras vasculares y nerviosas, como se muestra en los casos que presentamos, aunque también podemos mencionar la región del cuello, axila, articulación del codo y región inguinal. Al realizar este procedimiento no es necesario prolongar innecesariamente los brazos de la zetaplastia y con ello se consigue una ganancia longitudinal mucho mayor a una zetaplastia simple con despegamiento mínimo.

Es importante mencionar que los colgajos se deben levantar tan gruesos como sea posible; es decir, se deben levantar idealmente hasta el plano del tejido graso, teniendo cuidado de no lastimar elementos anatómicos infrayacentes (tendones, vasos, nervios, articulaciones). Al igual que otros autores, hemos observado que efectivamente se pueden levantar colgajos aun en zonas de quemadura o en zonas injertadas, logrando muy buenos resultados, tal como se reporta en la literatura. ${ }^{10,13,14}$ Se ha publicado recientemente otras técnicas de zetaplastias modificadas, como la zetaplastia de siete colgajos, con una mayor ganancia de elongación tisular. $^{15}$

La principal desventaja tal vez en este tipo de cirugías es que, en ocasiones, puede haber necrosis en el vértice de los colgajos, aunque sea parcial y ofrece poca liberación en los casos de bridas interdigitales, para los que la primera opción se pudiera realizar colgajos trapezoidales. ${ }^{16,17}$

\section{CONCLUSIÓN}

La utilización de la zetaplastia de cuatro colgajos es una técnica segura y fácil de reproducir, 
que corrige adecuadamente las bridas resultantes en los casos de quemadura. Su correcta planeación y realización evitará al paciente mayores cicatrices $y$, con despegamientos moderados, se minimiza el sangrado y lesiones estructurales, sobre todo en sitios anatómicos por donde discurren importantes elementos anatómicos.

\section{REFERENCIAS}

1. Secretaría de Salud. Vigilancia epidemiológica de las quemaduras. Sistema Único de Información. Boletín Epidemiológico del Sistema Nacional de Vigilancia Epidemiológica 2009; 25 (26).

2. Larson DL. The Prevention and correction of burn scar contracture and hypertrophy. Shriners Burns Institute. University of Texas Medical Branch. Galveston, Texas 1973.

3. Maillard GF, Clavel PR. Aesthetic units in skin grafting of the face. Ann Plast Surg 1991; 26 (4): 347-52.

4. Kryger ZB, Sisco M. The reconstructive ladder. In: Practical Plastic Surgery. Landes Bioscience (Vademecum) Austin, Texas USA; 2007, pp. 47-48.

5. Dhennin C. Surgery for sequelae of burns. Rev Prat 1995; 45 (5): 590-5.

6. Ivy R. Who originated the Z-plasty? Plast Reconstr Surg 1971; 47: 67-72.

7. Kryger ZB, Sisco M. Z-Plasty Basic concepts in wound repair. In: Practical Plastic Surgery. Austin, Texas USA: Landes Bioscience (Vademecum); 2007, pp. 9-10.

8. Trigos MI, Pérez GA. Malformaciones congénitas de los párpados. Propuesta de clasificación y manejo quirúrgico. Cir Plast 1997; 7 (3): 111-120.
9. Mir y Mir L. The six-flap z-plasty. Plast Reconstr Surg 1973; 52 (6): 625-628.

10. Barret JP, Herndon DN, McCauley RL, Barret JP. Use of previously burned skin as random cutaneous local flaps in pediatric burn reconstruction. Burns 2002; 28 (5): 500-2.

11. Scheider RM, Simonton TS. Tratamiento de articulaciones y escaras. En: Achauer B. Manejo del paciente quemado. México: El Manual Moderno; 1988, pp. 217.

12. Young RC, Burd A. Pediatric upper limb contracture release following burn injury. Burns 2004; 30 (7): 723 728.

13. Parry I, Kimberly W, Niszczak J, Palmieri T, Greenhalgh D. Methods and tools used for the measurement of burn scar contracture. J Burn Care Res 2010; 31 (6): 888-903.

14. Cenk Sen et al. A simple and effective procedure for treating burn contractures: Releasing incision and quadra Z technique. Burns 2007; 33 (2): 241-245.

15. Yilmaz $\mathrm{S}$ et al. The seven flap Z-plasty revised. Burns 2003; 29 (8): 849-853.

16. Grishkevich V. Trapezoid adipose scar local flap: post burn lateral truncal contracture elimination with Trapeze-flap Plasty. J Burn Care Res 2010; 31 (6): 949954.

17. Grishkevich V. First web space post-burn contracture types: contracture elimination methods. Burns 2011; 37 (2): 330-339.

Correspondencia:

Dr. José Luis Pérez Avalos.

Zacatecas 36-113, Col. Roma, 06700, Ciudad de México, México

E-mail: perezavalosmd@gmail.com perezavalos@correo.xoc.com.mx 